\title{
Opportunistic Scheduling of Control Tasks Over Shared Wireless Channels:
}

\author{
Konstantinos Gatsis, Miroslav Pajic, Alejandro Ribeiro, and George J. Pappas \\ Department of Electrical and Systems Engineering \\ University of Pennsylvania \\ 200 S. 33rd Street, Philadelphia, PA 19104 \\ \{kgatsis, pajic, aribeiro, pappasg\}@seas.upenn.edu
}

\begin{abstract}
We consider a wireless networked control system with multiple loops closing over a shared wireless medium. To avoid interferences a centralized scheduler decides which control task accesses the channel at each time step, opportunistically based on the random wireless channel conditions that systems experience. We formulate the problem of designing channel-aware scheduling and transmit power allocation mechanisms that guarantee Lyapunov-like performances for all control tasks in expectation over the channel conditions, while they also minimize the total power expenditures. Exploiting the zero duality gap, optimal variables are obtained by solving at the dual domain either offline, or online based on the observed random channel sequence. Simulations illustrate the power savings of the opportunistic scheme.
\end{abstract}

\section{Categories and Subject Descriptors}

I.2.8 [Artificial Intelligence]: Problem Solving, Control Methods, and Search—Control theory, Scheduling; C.2.1 [Computer Communication Networks]: Network Architecture and Design-Wireless communication; G.1.6 [Numerical Analysis]: Optimization-Stochastic programming

\section{General Terms}

Theory

\section{INTRODUCTION}

As the number of networked control systems (NCSs) applications increases in, e.g., smart building or industrial en-

\footnotetext{
*This work was supported in part by NSF CNS-0931239, and by TerraSwarm, one of six centers of STARnet, a Semiconductor Research Corporation program sponsored by MARCO and DARPA.
}

Permission to make digital or hard copies of all or part of this work for personal or classroom use is granted without fee provided that copies are not made or distributed for profit or commercial advantage and that copies bear this notice and the full citation on the first page. To copy otherwise, to republish, to post on servers or to redistribute to lists, requires prior specific permission and/or a fee.

Copyright 200X ACM X-XXXXX-XX-X/XX/XX ...\$5.00. vironments, the available communication and computation resources often need to be shared among different tasks. Efficient resource management in such setups is important to meet desirable stability and control performance requirements. In particular, when multiple control loops need to communicate over a shared medium or execute on a shared CPU, scheduling access to the resource becomes instrumental.

To analyze and design such scheduling mechanisms, previous works have related the resource utilization to some control performance metric or stability requirement, including, e.g., linear quadratic costs [3, 11], decrease rates of Lyapunov functions [5, 10], reachability and observability requirements [16], sampling rates, maximum allowed transmission intervals and delays [2]17], or automata executions [15]. Resource utilization on the other hand is generally expressed as the average resource time spent on each task - e.g., how often a task accesses a communication medium [11], or the ratio of execution time over each sampling period in a CPU [3]. The form of the proposed schedulers and the design methodologies vary depending on how this relationship between resource allocation and control requirement is expressed. The problem of control/scheduling co-design has also been examined (e.g. [11,16]), however this often results in a hard combinatorial optimization problem [13].

In this paper we are interested in the problem of scheduling control tasks over shared wireless communication mediums. While the above scheduling mechanisms are designed to meet control performance requirements, they lack awareness on the physical layer aspects of the problem. The channel conditions on the shared wireless medium not only change unpredictably over time but also differ among users. A channelaware scheduling mechanism has the potential to make communication within the loop more efficient by, e.g., opportunistically scheduling control tasks under favorable channel conditions, or equivalently preventing them from transmitting under adverse conditions. Such opportunistic resource allocation mechanisms have been developed in the context of multi-user wireless communication networks [8, 12, 14], where the objective is typically to maximize some user utility measure, e.g., communication rate. Additionally these mechanisms offer efficient utilization of the available com- 


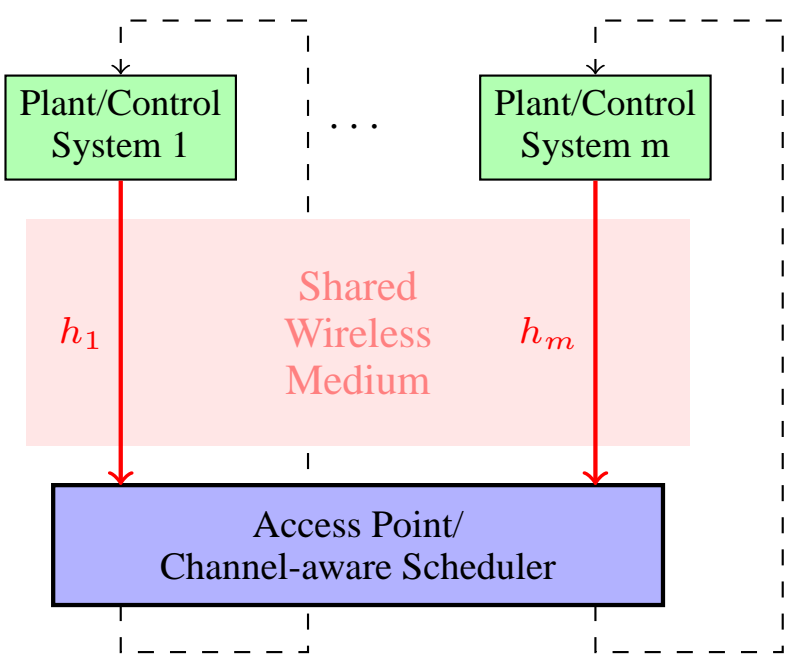

Figure 1: Opportunistic scheduling of control tasks over a shared wireless medium. Independent control systems close the loop by transmitting over the shared medium to a common receiver/access point. Each control system $i$ experiences random channel conditions $h_{i}$. A centralized scheduler located at the access point observes the vector of channel states $h$ and opportunistically decides which system is scheduled to transmit and close the loop.

munication power resources.

A proliferation of opportunistic resource allocation into wireless NCSs requires a suitable interface that would bridge control performance requirements with the wireless physical layer design aspects of the problem. In this paper we consider a set of independent NCSs closing their loops by transmitting over a shared wireless channel (see Fig. 1) and we abstract their control performance requirements as desired decrease rates of given Lyapunov functions (Section 2). When a system transmits, the probability of successful delivery at the receiver depends on the allocated transmit power as well as the current channel conditions, following a model examined in our previous work [6,7]. An opportunistic scheduler then allocates channel access among systems by observing the random channel conditions experienced by each of them, and needs to guarantee the prescribed Lyapunov decrease rates of each system in expectation over the possible channel states.

Furthermore, we formulate the design of channel-aware scheduling and transmit power allocation as a stochastic optimization problem in Section 2.1 where the objective is to minimize the expected total power expenditures subject to the expected Lyapunov decrease rate constraints. By exploiting the zero duality gap of the problem, in Section 3 we present a subgradient algorithm that solves the dual problem and we show how the optimal scheduling and power allocation can be derived from the dual optimal solution.

Implementing the subgradient algorithm on the other hand is computationally demanding and requires knowledge of the probability distribution of the channel states, which is not available in most practical setups. Hence we develop a stochastic subgradient algorithm in Section 4 which utilizes the observed channel sequence during execution to solve the dual problem online. Moreover we establish in Theorem 1 that if the scheduling and power allocation variables are selected online according to the algorithm, the required Lyapunov performance constraints are met in the limit in a strong sense (almost surely). Finally, we present simulations of the proposed design methodology in Section 5 and we observe a significant reduction to the magnitude of $80 \%$ in the power consumption compared to non-channel-aware mechanisms for a simple wireless NCS example. We conclude with a discussion on our results in Section 6

Notation: We denote the real $m$-dimensional non-negative orthant with $\mathbb{R}_{+}^{m}$, and the element-wise comparison with respect to the orthant by $\geq$, i.e., $x \geq y$ if and only if $x-y \in$ $\mathbb{R}_{+}^{m}$. The set of $n \times n$ real symmetric positive semi-definite (respectively definite) matrices is denoted by $S_{+}^{n}$ (respectively $S_{++}^{n}$ ), and with $\succeq$ the comparison with respect to the cone $S_{+}^{n}$. We denote the $m$-dimensional probability simplex by $\Delta^{m}$, i.e., $\Delta^{m}=\left\{\alpha \geq 0: \sum_{i} \alpha_{i} \leq 1\right\}$.

\section{PROBLEM DESCRIPTION}

Consider the wireless control architecture of Fig. 1 consisting of $m$ independent networked control systems. At each time $k$, by $x_{i, k} \in \mathbb{R}^{n_{i}}$ we denote the state of the system $i(i=1,2, \ldots, m)$. To keep the framework general we assume that different descriptions of the system evolution from $x_{i, k}$ to $x_{i, k+1}$ at time $k$ are given depending on whether a transmission occurs at time $k$ or not. Let us indicate with $\gamma_{i, k} \in\{0,1\}$ the event that such a transmission occurs at time $k$ for the subsystem $i$. For simplicity then we describe the system evolution by a linear time invariant model,

$$
x_{i, k+1}=\left\{\begin{array}{ll}
A_{c, i} x_{i, k}+w_{i, k}, & \text { if } \gamma_{i, k}=1 \\
A_{o, i} x_{i, k}+w_{i, k}, & \text { if } \gamma_{i, k}=0
\end{array} .\right.
$$

At a successful transmission the system dynamics are described by the matrix $A_{c, i} \in \mathbb{R}^{n_{i} \times n_{i}}$, where 'c' stands for closed-loop, and otherwise by $A_{o, i} \in \mathbb{R}^{n_{i} \times n_{i}}$, where 'o' stands for open-loop. We assume that $A_{c, i}$ is asymptotically stable, implying that if system $i$ were to transmit at each slot its respective state evolution is stable. The open loop matrix $A_{o, i}$ could be unstable. The additive terms $w_{i, k}$ model an independent identically distributed (i.i.d.) noise process with mean zero and covariance $W_{i} \succeq 0$. It is worth noting that closed-loop dynamics for all of the $m$ controllers are fixed (meaning that adequate controllers have been already designed), and thus in this work we focus on designing the wireless communication aspects of system.

Example 1. The above networked control system description models various control architectures. For example sup- 
pose each closed loop $i$ consists of a linear plant of the form

$$
x_{i, k+1}=A_{i} x_{i, k}+B_{i} u_{i, k}+w_{i, k},
$$

and a wireless sensor transmitting the plant state measurement $x_{i, k}$ to a controller/actuator which provides input $u_{i, k}$. Let then the controller apply a linear feedback $u_{i, k}=K_{i} x_{i, k}$ when a measurement is received, otherwise apply $u_{i, k}=0$. The resulting closed loop is of the form (1) with $A_{c, i}=$ $A_{i}+B_{i} K_{i}$ and $A_{o, i}=A_{i}$. Dynamic controllers, e.g., using some local plant state estimate when packets are dropped, can also be expressed in form (1) by enlarging the state of the overall closed loop system to contain both plant and controller states.

We now describe the wireless communication system and model how it determines the packet transmission indicators $\gamma_{i, k}$. We describe the wireless channel conditions for a system $i$ at time $k$ by the channel fading coefficient $h_{i, k}$ that system $i$ experiences if it transmits at time $k$. Due to propagation effects the channel gains $h_{i, k}$ change unpredictably [9, Ch. 3] and take values in a subset $\mathcal{H} \subseteq \mathbb{R}_{+}$of the positive reals. Channel states $h_{i, k}$ for all systems $1 \leq i \leq m$ are grouped in a vector $h_{k} \in \mathcal{H}^{m}$. We adopt a block fading model whereby channel states $h_{k}$ are modeled as random variables independent across different time slots $k$ and identically distributed according to some joint distribution $\phi$ on $\mathcal{H}^{m}$. They are also independent of the plant process noise $w_{i, k}$. We assume the channel states are available before transmission - see Remark 1 for a discussion on practical implementation. We also make the following technical assumption on their joint distribution to avoid degeneracies.

ASSUMPTION 1. The joint distribution $\phi$ of channel states $h_{k}$ has a probability density function on $\mathcal{H}^{m}$.

If system $i$ transmits at time $k$ a transmit power level $p_{i, k}$ taking values in $0 \leq p_{i, k} \leq p_{\max }$ is selected. Then channel fading and transmit power affect the probability of successful decoding of the transmitted packet at the receiver. In particular given the forward error-correcting code (FEC) in use, the probability $q$ that a packet is successfully decoded is a function of the received signal-to-noise ratio (SNR). The SNR is proportional to the received power level expressed by the product $h p$ of the channel fading state and the allocated transmit power. Overall we express the probability of success by some given relationship of the form $q\left(h_{i, k}, p_{i, k}\right)$ (see, e.g., [7] for more details on this model). An illustration of this relationship is shown in Fig. 2. The following assumption on the form of the function $q(h p)$ will be helpful in the subsequent sections.

ASSUMPTION 2. The function $q($.$) as a function of the$ product $r=h$ for $r \geq 0$ satisfies:

(a) $q(0)=0$,

(b) $q(r)$ is continuous, and strictly increasing when $q(r)>$ 0 , i.e., for any $r^{\prime}>r$ it holds that $q\left(r^{\prime}\right)>q(r)$,

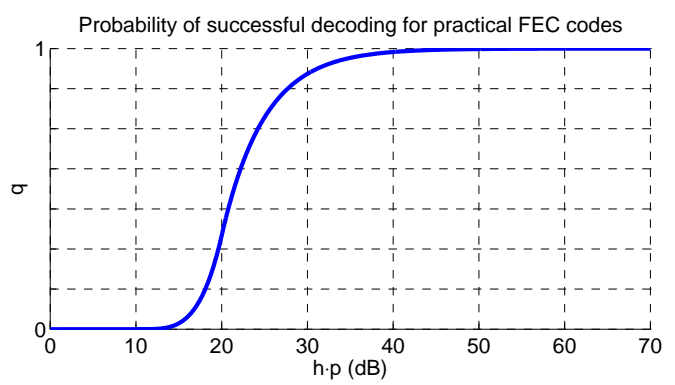

Figure 2: Complementary error function for practical FEC codes. The probability of successful decoding $q$ for a FEC code is a sigmoid function of the received $\mathbf{S N R} \sim h p$.

(c) for any $\mu \geq 0$ and for almost all values $h \in \mathcal{H}$ the set $\operatorname{argmin}_{0 \leq p \leq p_{\max }} p-\mu q(h p)$ is a singleton.

Parts (a),(b) of this assumption state that the probability of successful decoding $q(h p)$ will be zero when the received power level $h p$ is small, and it becomes positive $q(h p)>0$ and strictly increasing for larger values of $h p$. Part (c) is more stringent, stating essentially that $q(h p)$ cannot behave linearly in $p$ for a range of channel values $h$. As shown in Fig. 2 for cases of practical interest $q(h p)$ has a sigmoid form and all the above requirements are expected to hold.

Apart from packet drops due to low received SNR, packet collisions may occur if more than one of the control systems transmit at a given time slot over the shared wireless medium. For this reason we are interested in designing a mechanism to select which system will access the channel, i.e., which system is scheduled to transmit. We allow for a randomized selection, hence let us denote with $\alpha_{i, k}$ the probability of scheduling system $i$ at time $k$. The scheduling variables satisfy

$$
\alpha_{i, k} \geq 0, \sum_{i=1}^{m} \alpha_{i, k} \leq 1,
$$

or in other words $\alpha_{k}$ belongs in the probability simplex $\Delta^{m}$. Note that this implicitly models a centralized scheduling mechanism that by design avoids packet collisions since at most one plant is scheduled to transmit.

To sum up, we can model transmission success $\gamma_{i, k}$ given scheduling variables, power allocation, and channel state, as Bernoulli random variables with success probability

$$
\mathbb{P}\left[\gamma_{i, k}=1 \mid h_{i, k}, \alpha_{i, k}, p_{i, k}\right]=\alpha_{i, k} q\left(h_{i, k}, p_{i, k}\right)
$$

This expression states that the probability that system $i$ successfully transmits and closes the loop equals the probability that $i$ is scheduled to transmit, multiplied by the probability that the message is correctly decoded. For compactness we group scheduling decisions and power allocations of all systems $i$ at time $k$ into vectors $\alpha_{k} \in \Delta^{m}$, and $p_{k} \in\left[0, p_{\max }\right]^{m}$ respectively. 
Our goal is to design the wireless communication variables of the system opportunistically, that is to select appropriate scheduling and power allocation variables adapting to the current wireless channel conditions described by $h_{k}$. Overall we express the scheduling and power decisions $\alpha_{k}, p_{k}$ respectively as mappings of the form

$$
\mathcal{A}=\left\{\alpha: \mathcal{H}^{m} \mapsto \Delta^{m}\right\}, \quad \mathcal{P}=\left\{p: \mathcal{H}^{m} \mapsto\left[0, p_{\text {max }}\right]^{m}\right\}
$$

so that $\alpha_{k}=\alpha\left(h_{k}\right), p_{k}=p\left(h_{k}\right)$. Since channel states $h_{k}$ are independent over time $k$ these mappings do not need to change over time. Substituting the scheduling and power allocation mappings $\alpha(),. p($.$) in our communication model$ described by (4) the probability of successful transmission for each system $i$ at any given slot $k$ becomes

$$
\begin{aligned}
\mathbb{P}\left(\gamma_{i, k}=1\right) & =\mathbb{E}_{h_{k}}\left\{\mathbb{P}\left[\gamma_{i, k}=1 \mid h_{i, k}, \alpha_{i}\left(h_{k}\right), p_{i}\left(h_{k}\right)\right]\right\} \\
& =\mathbb{E}_{h} \alpha_{i}(h) q\left(h_{i}, p_{i}(h)\right) .
\end{aligned}
$$

Here the expectation is with respect to the joint distribution $\phi$ of the vector channel realization $h_{k}$ which we assumed to be identical for any time $k$, hence we dropped the index $k$. Note also that the communication process modeled by the sequence $\left\{\gamma_{i, k}, 1 \leq i \leq m, k \geq 0\right\}$ depends only on variables related to the wireless communication counterpart of the overall system, and is in particular independent of the system states $x_{i, k}$.

Our primary goal in designing the communication variables of the system is to guarantee a level of closed loop performance for each subsystem. To motivate a more formal problem description we consider Lyapunov-like performance requirements for the control systems. In particular suppose quadratic Lyapunov functions of the form $V_{i}(x)=$ $x^{T} P_{i} x, x \in \mathbb{R}^{n_{i}}$, with $P_{i} \in S_{++}^{n_{i}}$, are given for each system $i$. A Lyapunov-like requirement then states that these functions should decrease at given rates $\rho_{i}<1$ during the execution of each subsystem $i$. This evolution however is random because of the stochastic nature of the overall wireless communication/control system, i.e., due to process noise, random channel states, randomized channel access, and packet drops. To take these effects into account we require that at any value $x_{i, k} \in \mathbb{R}^{n_{i}}$ of system state $i$ at time $k$ the Lyapunov functions at the next time step decrease at the desired rate $\rho_{i}<1$ in expectation, that is

$$
\mathbb{E}\left[V_{i}\left(x_{i, k+1}\right) \mid x_{i, k}\right] \leq \rho_{i} V_{i}\left(x_{i, k}\right)+\operatorname{Tr}\left(P_{i} W_{i}\right) .
$$

The expectation over the next system state $x_{i, k+1}$ on the left hand side accounts via (1) for the randomness introduced by the process noise $w_{i, k}$ as well as the transmission success $\gamma_{i, k}$. The effect of process noise appears on the right hand side as the constant term $\operatorname{Tr}\left(P_{i} W_{i}\right)$, while the transmission success is expressed in (6) and depends on the observed channel state $h_{k}$ as well as the communication decisions $\alpha_{k}, p_{k}$. The intuition behind requirement (7) is that if it holds on each time $k$ it follows that

$$
\mathbb{E}\left[V_{i}\left(x_{i, N}\right) \mid x_{i, 0}\right] \leq \rho_{i}^{N} V_{i}\left(x_{i, 0}\right)+\frac{1-\rho_{i}^{N}}{1-\rho_{i}} \operatorname{Tr}\left(P_{i} W_{i}\right)
$$

meaning that system states have second moments that decay exponentially and in the limit remain bounded by the constant $\operatorname{Tr}\left(P_{i} W_{i}\right) /\left(1-\rho_{i}\right)$.

On the other hand, apart from control performance requirements an efficient communication design should make an efficient use of the available power resources at the devices. The induced overall expected power consumption on each slot $k$ is given by

$$
\mathbb{E}_{h_{k}} \sum_{i=1}^{m} \alpha_{i, k}\left(h_{k}\right) p_{i, k}\left(h_{k}\right)
$$

summing up the transmit power of each system $i$ if the system is scheduled to transmit. The expectation here is with respect to the joint channel distribution $h_{k} \sim \phi$. The approach we take in designing scheduling and power allocation (cf. (5)] that are control-performance aware (cf (7) and also energy-efficient (cf. (9) is through a stochastic optimization framework that we present next.

Remark 1. The centralized channel-aware scheduler can be implemented in a multiple access channel architecture as shown in Fig. 1, where each control system transmits to a common access point. For example each plant state is measured by a sensor, and all sensors transmit plant measurements to a centralized controller responsible for providing plant inputs at each plant. The channel conditions for each system can be measured at the access point by pilot signals sent from the sensors to the access point. Depending on the measured channel states the access point decides which plant is scheduled to close the loop.

\subsection{Scheduling and power allocation as stochas- tic optimization}

We pose the problem of designing the scheduling and power allocation mappings $\alpha(),. p($.$) as an optimization problem$ where at each slot we minimize the total expected power consumption (9) of the design while satisfying the Lyapunov requirements (7), i.e.,

$$
\begin{array}{ll}
\underset{\alpha \in \mathcal{A}, p \in \mathcal{P}}{\operatorname{minimize}} & \mathbb{E}_{h_{k}} \sum_{i=1}^{m} \alpha_{i}\left(h_{k}\right) p_{i}\left(h_{k}\right) \\
\text { subject to } & \mathbb{E}\left[V_{i}\left(x_{i, k+1}\right) \mid x_{i, k}\right] \leq \rho_{i} V_{i}\left(x_{i, k}\right)+\operatorname{Tr}\left(P_{i} W_{i}\right) \\
& \text { for all } x_{i, k} \in \mathbb{R}^{n_{i}}, i=1, \ldots, m
\end{array}
$$

To make explicit how the functions $\alpha(),. p($.$) appear in the$ constraints observe that by (1) we have

$$
\begin{aligned}
& \mathbb{E}\left[V_{i}\left(x_{i, k+1}\right) \mid x_{i, k}\right]=\mathbb{P}\left(\gamma_{i, k}=1\right) x_{i, k}^{T} A_{c, i}^{T} P_{i} A_{c, i} x_{i, k} \\
& +\mathbb{P}\left(\gamma_{i, k}=0\right) x_{i, k}^{T} A_{o, i}^{T} P_{i} A_{o, i} x_{i, k}+\operatorname{Tr}\left(P_{i} W_{i}\right),
\end{aligned}
$$

where we used the fact that the random variable $\gamma_{i, k}$ is independent of the system state $x_{i, k}$ as it depends only on the 
communication variables (cf. (4) (6). Plugging (12) at the left hand side of the constraints in (10) we get

$$
\begin{aligned}
& \left(1-\mathbb{P}\left(\gamma_{i}=1\right)\right) x_{i, k}^{T} A_{o, i}^{T} P_{i} A_{o, i} x_{i, k} \\
& +\mathbb{P}\left(\gamma_{i, k}=1\right) x_{i, k}^{T} A_{c, i}^{T} P_{i} A_{c, i} x_{i, k} \leq \rho_{i} x_{i, k}^{T} P_{i} x_{i, k} .
\end{aligned}
$$

The decision variable in this constraint is the scalar $\mathbb{P}\left(\gamma_{i}=\right.$ 1) which depends on $\alpha(),. p($.$) by (6) Note that we may$ assume (13) holds when $\mathbb{P}\left(\gamma_{i}=1\right)=1$. This corresponds to the ideal case that system $i$ closes the loop on each slot (cf. (1) and we have assumed that $A_{c, i}$ is stable, so we may take the matrix $P_{i}$ to satisfy the control requirement $A_{c, i}^{T} P_{i} A_{c, i} \preceq$ $\rho_{i} P_{i}$ in this case.

Moreover the left hand side of (13) is a linear function of $\theta=\mathbb{P}\left(\gamma_{i, k}=1\right)$ at any $x_{i, k}$, hence if it holds for some $\theta_{0}$ it holds for the whole interval $\theta_{0} \leq \theta \leq 1$, as we assumed that it also holds for $\theta=1$. Finally observe that (13) according to problem (10) needs to hold at any value of $x_{i, k} \in \mathbb{R}^{n_{i}}$. Combining these observations, constraint (13) can be equivalently written as $c_{i} \leq \mathbb{P}\left(\gamma_{i}=1\right)$ where

$$
\begin{gathered}
c_{i}=\min \left\{\theta \geq 0: y^{T}\left[\theta A_{c, i}^{T} P_{i} A_{c, i}+(1-\theta) A_{o, i}^{T} P_{i} A_{o, i}\right] y\right. \\
\left.\leq \rho_{i} y^{T} P_{i} y \quad \text { for all } y \in \mathbb{R}^{n_{i}}\right\}
\end{gathered}
$$

Computing $c_{i}$ is a simple semidefinite optimization program which can be easily solved using available convex optimization software. The value $c_{i}$ represents the minimum required probability of transmission for each system $i$ that guarantees the desired Lyapunov decay rate $\rho_{i}$. Alternatively it can be thought of as a minimum required utilization factor of the shared wireless channel, analogously to a utilization of a shared CPU in, e.g., [3]. Intuitively, large value of $c_{i}$ implies that system $i$ requires more resources, i.e., more frequent channel access and possibly higher power expenditures.

To sum up, the Lyapunov constraints in problem (10) can be simplified by the auxiliary problems (14) for each $i$, so that (10) equivalently becomes

$$
\begin{array}{ll}
\underset{\alpha \in \mathcal{A}, p \in \mathcal{P}}{\operatorname{minimize}} & \mathbb{E}_{h} \sum_{i=1}^{m} \alpha_{i}(h) p_{i}(h) \\
\text { subject to } & c_{i} \leq \mathbb{E}_{h} \alpha_{i}(h) q\left(h_{i}, p_{i}(h)\right), i=1, \ldots, m
\end{array}
$$

This is the optimal scheduling and power allocation problem that we examine in this paper. Before proceeding to solve this problem let us make a final constraint qualification assumption that is typical in optimization theory, i.e., that a strictly feasible point exists.

Assumption 3. There exist variables $\alpha^{\prime} \in \mathcal{A}$ and $p^{\prime} \in$ $\mathcal{P}$ that satisfy the constraints of the optimization problem (15) with strict inequality, i.e.,

$$
c_{i}<\mathbb{E}_{h} \alpha_{i}^{\prime}(h) q\left(h_{i}, p_{i}^{\prime}(h)\right), \quad i=1, \ldots, m
$$

Since the problem $(15)$ is feasible we denote the optimal value with $P$ and an optimal solution with $\alpha^{*}(),. p^{*}($.$) . Sim-$ ilar optimization problems in the context of wireless communication networks have been considered in [14] where it is shown that they have zero duality gap. This fact will be exploited in the following sections to solve the problem of designing optimal scheduling and power allocation policies.

\section{OPTIMAL SCHEDULING AND POWER ALLOCATION}

In this section we exploit the fact that the problem of designing optimal channel-dependent scheduling and power allocation variables has zero duality gap. In particular we solve the problem in the dual domain using a subgradient method, and once the optimal dual variables are obtained we describe how the optimal variables of the original problem can be recovered.

First to derive the Lagrange dual problem of (15) consider non-negative dual variables $\mu \in \mathbb{R}_{+}^{m}$ corresponding to each of the $m$ constraints of (15) The Lagrangian is written

$$
\begin{aligned}
L(\alpha, p, \mu) & =\mathbb{E}_{h} \sum_{i=1}^{m} \alpha_{i}(h) p_{i}(h) \\
& +\sum_{i=1}^{m} \mu_{i}\left[c_{i}-\mathbb{E}_{h} \alpha_{i}(h) q\left(h_{i}, p_{i}(h)\right)\right],
\end{aligned}
$$

while the dual function is defined as

$$
g(\mu)=\min _{\alpha \in \mathcal{A}, p \in \mathcal{P}} L(\alpha, p, \mu) .
$$

For convenience let us also denote the set of pairs of functions $\alpha, p$ that minimize the Lagrangian at $\mu$ by

$$
(\mathcal{A}, \mathcal{P})(\mu)=\underset{\alpha \in \mathcal{A}, p \in \mathcal{P}}{\operatorname{argmin}} L(\alpha, p, \mu),
$$

whenever such solutions exist. In general this set might contain multiple solutions. We will refer to any such solution pair as $\alpha(\mu), p(\mu)$. We define then the Lagrange dual problem as follows.

$$
D=\underset{\mu \in \mathbb{R}_{+}^{m}}{\operatorname{maximize}} g(\mu) .
$$

The standard Lagrange duality theory informs us that the dual function $g(\mu)$ is a lower bound on the optimal cost $P$ of problem (15) for any $\mu$, so that the dual optimal value also satisfies $D \leq P$ (weak duality). The following proposition however establishes a strong duality result $(D=P)$ for the problem under consideration and provides a relationship between the optimal primal and dual variables.

Proposition 1. Let Assumptions 1 and 3 hold. Let $P$ be the optimal value of the optimization problem (15) and $\left(\alpha^{*}, p^{*}\right)$ be an optimal solution, and let $D$ be the optimal value of the dual problem (21) and $\mu^{*}$ be an optimal solution. Then

(a) $P=D$ (strong duality)

(b) $\mu_{i}^{*}\left[c_{i}-\mathbb{E}_{h} \alpha_{i}^{*}(h) q\left(h_{i}, p_{i}^{*}(h)\right)\right]=0$ for $i=1, \ldots, m$ (complementary slackness)

(c) $\left(\alpha^{*}, p^{*}\right) \in(\mathcal{A}, \mathcal{P})\left(\mu^{*}\right)$ 
Proof. Statement (a) under assumptions 1 and 3 follows immediately from [14, Theorem 1] where a similar optimization setup is examined. The proof is omitted due to space limitations.

To show (b) and (c) consider a primal optimal solution $\left(\alpha^{*}, p^{*}\right)$. This gives an optimal value $P$ for problem (15) It is also feasible, so the constraint slack given in the brackets of part (b), which we will denote as $s_{i}$ for compactness within this proof, is non-positive. The Lagrangian then given in (18) evaluates to

$$
L\left(\alpha^{*}, p^{*}, \mu^{*}\right)=P+\sum_{i} \mu_{i}^{*} s_{i} \leq P,
$$

where the last inequality follows because $s_{i} \leq 0$ and $\mu_{i}^{*} \geq 0$.

On the other hand by definition of the dual function $g(\mu)$ in (19) at $\mu^{*}$ we have that

$$
L\left(\alpha^{*}, p^{*}, \mu^{*}\right) \geq g\left(\mu^{*}\right)=P
$$

where we used that $g\left(\mu^{*}\right)=D=P$ by part (a) at the left hand side of (23) Combining (22) and (23) we conclude that all the included inequalities hold with equality. This means that $\sum_{i} \mu_{i}^{*} s_{i}=0$, which is part (b), and also that $\alpha^{*}, p^{*}$ are Lagrangian optimizers at $\mu^{*}$, which verifies (c).

Interestingly this proposition states that strong duality holds regardless of the form of function $q(h, p)$ appearing in the constraints (note that we have not enforced Assumption 2 yet). More importantly, as we follow next, it suggests the possibility of solving first the dual problem instead of the primal, find the optimal dual variables $\mu^{*}$, and via (c) attempt to recover the optimal primal variables $\alpha^{*}, p^{*}$.

To maximize the dual function $g(\mu)$ for the dual problem (21) we employ a dual projected subgradient algorithm [1] Ch. 8]. A subgradient direction $s(\mu) \in \mathbb{R}^{m}$ for the (concave) function $g(\mu)$ with respect to $\mu \in \mathbb{R}_{+}^{m}$ is a vector that satisfies

$$
g\left(\mu^{\prime}\right)-g(\mu) \leq\left(\mu^{\prime}-\mu\right)^{T} s(\mu) \quad \text { for all } \mu^{\prime} \in \mathbb{R}_{+}^{m} .
$$

If we pick $\alpha(\mu), p(\mu) \in(\mathcal{A}, \mathcal{P})(\mu)$ by $(20)$ then a subgradient $s(\mu)$ can be found as the constraint slack of the primal problem (15) evaluated at these points,

$$
s_{i}(\mu)=c_{i}-\mathbb{E}_{h} \alpha_{i}(\mu ; h) q\left(h_{i}, p_{i}(\mu ; h)\right) .
$$

To show this observe that for any other $\mu^{\prime}$ in general we have $g\left(\mu^{\prime}\right) \leq L\left(\alpha(\mu), p(\mu), \mu^{\prime}\right)$. Subtracting from each side of this inequality the term $g(\mu)=L(\alpha(\mu), p(\mu), \mu)$ and expanding the terms of the Lagrangian as in (18) we get

$g\left(\mu^{\prime}\right)-g(\mu) \leq \sum_{i=1}^{m}\left(\mu_{i}^{\prime}-\mu_{i}\right)\left[c_{i}-\mathbb{E}_{h} \alpha_{i}(\mu ; h) q\left(h_{i}, p_{i}(\mu ; h)\right)\right]$,

which is exactly the definition of the subgradient in (24). We also note for future reference that for any $\mu$ the subgradients $s(\mu)$ are bounded because at the right hand side of (25) the term $c_{i}$ is bounded (cf (14) and the term in the expectation corresponds to a probability (cf (6) .
A projected dual subgradient ascent method to maximize the concave dual function $g(\mu)$ is described as follows:

1. At iteration $t$ given $\mu(t)$ compute primal optimizers of the Lagrangian at $\mu(t)$

$$
p(.)(t), \alpha(.)(t) \in(\mathcal{A}, \mathcal{P})(\mu(t))
$$

2. Evaluate the subgradient vector $s(\mu(t))$ by (25)] and update the dual variables by an ascent step

$$
\mu(t+1)=[\mu(t)+\epsilon(t) s(\mu(t))]_{+}
$$

where $[\quad]_{+}$denotes the projection on the non-negative orthant and $\epsilon(t)>0$ is the stepsize.

It is well established that the above algorithm converges to an optimal solution $\mu^{*}$ for stepsizes that are square summable but not summable, i.e.,

$$
\sum_{t=1}^{\infty} \epsilon(t)^{2}<\infty, \sum_{t=1}^{\infty} \epsilon(t)=\infty
$$

In that case we have that $\mu(t) \rightarrow \mu^{*}$ and $g(\mu(t)) \rightarrow g\left(\mu^{*}\right)=$ $D=P$. For a proof see, e.g., [1, Prop. 8.2.6].

In order to implement the subgradient algorithm above we need an efficient way to compute primal Lagrange optimizers in (27) solving (20) Note that this problem also relates to our capability of finding the optimal primal variables of interest $\alpha^{*}, p^{*}$ as we have shown in Proposition 1(c). Hence we turn our focus to problem (20). A more convenient expression for the Lagrangian defined in (18) can be obtained by rearranging terms to get

$$
L(\alpha, p, \mu)=\mu^{T} c+\mathbb{E}_{h} \sum_{i=1}^{m} \alpha_{i}(h)\left[p_{i}(h)-\mu_{i} q\left(h_{i}, p_{i}(h)\right)\right] .
$$

This form provides a useful separation of the primal variables first across channel realizations $h$, and second across systems $i$. We exploit this structure in the following lemma to design primal Lagrangian optimizers.

Lemma 1. Solutions $\alpha(\mu), p(\mu) \in(\mathcal{A}, \mathcal{P})(\mu)$ of problem (20) for any $\mu \in \mathbb{R}_{+}^{m}$ can be obtained at each $h \in \mathcal{H}^{m}$ as:

$$
p_{i}(\mu ; h)=p_{i}\left(\mu_{i} ; h_{i}\right)=\underset{0 \leq p \leq p_{\max }}{\operatorname{argmin}} p-\mu_{i} q\left(h_{i}, p\right),
$$

and

$$
\alpha(\mu ; h)=\underset{\alpha \in \Delta^{m}}{\operatorname{argmin}} \sum_{i=1}^{m} \alpha_{i} \xi\left(h_{i}, \mu_{i}\right)
$$

where

$$
\xi\left(h_{i}, \mu_{i}\right)=\min _{0 \leq p \leq p_{\max }} p-\mu_{i} q\left(h_{i}, p\right) .
$$

Moreover if Assumptions 1 and 2 hold, then for any $\mu \in \mathbb{R}_{+}^{m}$ and $\alpha(\mu), p(\mu) \in(\mathcal{A}, \mathcal{P})(\mu)$ the vector $s(\mu)$ defined in $(25)$ has a unique value. 
Proof. See Appendix A.1

The first clause of the lemma provides through equations (31) and (32) a method to obtain primal Lagrange optimizers that can be used in step (25) of the subgradient algorithm. Additionally an interesting separability result for the power allocation across systems $i$ is revealed - see Remark 2.

The second clause of the lemma states that under Assumptions 1 and 2 for any dual variable $\mu \in \mathbb{R}_{+}^{m}$ the subgradient vector $s(\mu)$ in (25), which is also the constraint slack of any Lagrange optimizers $\alpha(\mu), p(\mu)$ of (15) is unique. Consider then the case for the optimal dual variable $\mu^{*}$. By Prop. 1 (c) the optimal primal variables $\alpha^{*}, p^{*}$ are Lagrange optimizers at $\mu^{*}$, and since they are feasible they have a non-positive slack $s\left(\mu^{*}\right) \leq 0$. Hence by the above lemma we conclude that all Lagrange optimizers $\alpha\left(\mu^{*}\right), p\left(\mu^{*}\right)$ at $\mu^{*}$ have the same non-positive slack, i.e., they are feasible for the primal problem, and they also have the same objective value for the primal problem as $\alpha^{*}, p^{*}$, hence they are optimal too. This, along with the subgradient algorithm to find $\mu^{*}$, completes the method for solving the optimal scheduling and power allocation problem (15) We summarize these findings in the following proposition.

Proposition 2. Consider the optimization problem (15) and its dual derived in (21) and let Assumptions 1,2$] 3$ hold. Then the iterates $\mu(t)$ of the algorithm (27) (28) with stepsizes (29) converge to an optimal dual solution $\mu^{*}$, and optimal primal solutions $\alpha^{*}, p^{*}$ can be obtained by solving (31). (33) at the point $\mu^{*}$.

Motivated by the presented offline methodology to solve for the optimal scheduling and power allocation, in the next section we develop an online algorithm to solve the same problem by observing the sequence of channel realizations during system execution.

Remark 2. The optimal scheduling and power allocation mappings $\alpha^{*}, p^{*}$ according to Prop 2 can be obtained by solving (31) (33) at the point $\mu^{*}$. In particular, given $\mu^{*}$ and channel states $h$ the optimal power allocation $p_{i}^{*}(h)$ by (31) depends only on the variables $\mu_{i}, h_{i}$ pertinent to system $i$ and not on the whole vectors $\mu^{*}$ or $h$. Hence power allocation exhibits a completely decentralized structure, which we have made explicit in (31) by denoting it as $p_{i}\left(\mu_{i} ; h_{i}\right)$. Similar separability results are also known in the context of wireless communication networks [14]. Note however that the optimal scheduling in (32) is centralized since it depends on the whole vector $\mu^{*}$ and the channel realizations $h$ of all systems. Finally observe that the optimal scheduling is to select the system $i$ with the minimum value $\xi\left(h_{i}, \mu_{i}^{*}\right)$, i.e., scheduling is in general deterministic given the channel observation $h$. Randomization is introduced by the stochasticity of the channel realization.

\section{STOCHASTIC ONLINE SCHEDULING AND POWER ALLOCATION}

Implementing the subgradient algorithm presented in the previous section to solve for the optimal scheduling and power allocation is challenging in practice. In the primal step (27) one needs to solve for $\alpha(h), p(h)$ for a continuum of variables $h \in \mathcal{H}^{m}$, while for the dual step in (28) one needs to compute the subgradient direction $s(\mu)$ in (25) by integrating over the channel distribution $\phi$. A practical implementation would require drawing a sufficiently large number of samples from $\phi$ and solving for primal variables at these samples to obtain an estimate of the actual subgradient direction. This in turn is computationally intensive, does not scale for a large number of systems $m$, while also in most cases of practical interest the channel distribution is not available. These drawbacks motivate us to develop an online algorithm.

The algorithm we propose is a stochastic version of the primal/dual steps (27), (28) of the subgradient method of the previous section and does not rely on knowledge of the channel distribution. It is instead based only on the observed channel realization $h_{k}$ at each step. The proposed algorithm is summarized as follows.

1. At time slot $k$ observe the channel realization $h_{k}$, and given the variables $\mu_{k}$ compute $\alpha_{k}, p_{k}$ as primal Lagrangian optimizers at $h_{k}$ by (31) (32), i.e.,

$$
\begin{aligned}
p_{i, k} & =p_{i}\left(\mu_{i, k} ; h_{i, k}\right), \quad i=1, \ldots, m, \\
\alpha_{k} & =\alpha\left(\mu_{k} ; h_{k}\right)
\end{aligned}
$$

2. Compute the vector

$$
s_{i, k}=c_{i}-\alpha_{i, k} q\left(h_{i, k}, p_{i, k}\right), \quad i=1, \ldots, m,
$$

and update the variables $\mu_{k}$ by

$$
\mu_{k+1}=\left[\mu_{k}+\epsilon_{k} s_{k}\right]_{+} .
$$

where []$_{+}$denotes the projection on the non-negative orthant and $\epsilon_{k}>0$ is the stepsize.

To emphasize that this is an online algorithm we have explicitly indexed the variables with $k$ corresponding to real time slots. This algorithm gives a sequence of scheduling and power variables $\left\{\alpha_{k}, p_{k}, k \geq 0\right\}$ as well as (dual) variables $\left\{\mu_{k}, k \geq 0\right\}$ which are random because they depend on the random observed sequence of channel realizations $\left\{h_{k}, k \geq 0\right\}$. The main difference compared to the subgradient algorithm of the previous section is that it follows random directions $s_{k}$ by (35) instead of the exact subgradient directions $s\left(\mu_{k}\right)$ by (25) Comparing these two expressions it is immediate that the expected value of $s_{k}$ coincides with the subgradient $s\left(\mu_{k}\right)$, so it is reasonable to conjecture that the online algorithm is expected to move towards the maximum of the dual function, as the subgradient method does. The following proposition indeed establishes convergence in a strong sense.

PRoposition 3. Consider the optimization problem (15) and its dual derived in (21) and let Assumption 3 hold. Based 
on a sequence $\left\{h_{k}, k \geq 0\right\}$ of i.i.d. random variables with distribution $\phi$, let the algorithm described in steps (34) (36) be employed using stepsizes satisfying (29). Then almost surely we have that

$$
\lim _{k \rightarrow \infty} \mu_{k}=\mu^{*}, \text { and } \lim _{k \rightarrow \infty} g\left(\mu_{k}\right)=D
$$

where $\mu^{*}$ is an optimal solution of the dual problem and $D$ is the optimal value of the dual problem.

\section{Proof. See Appendix A.2}

The proposition states that the stochastic online algorithm yields a random sequence of dual variables $\mu_{k}$ that converge to the optimal dual variables $\mu^{*}$ in a strong sense, i.e., almost surely for any sequence of channel realizations that is observed. However the original problem of interest as expressed in (10) is that of selecting scheduling and power allocation policies that satisfy the given Lyapunov performance requirements (7). Hence the following Theorem characterizes the performance of the control systems if the communication variables are selected online according to the proposed algorithm.

THEOREM 1. Consider a shared wireless control architecture composed of $m$ systems described by (1) communication modeled by (4) depending on channel states $h_{k}$ which are i.i.d. with distribution $\phi$, as well as on scheduling and power allocation variables $\alpha_{k}, p_{k}$ chosen according to the algorithm described by steps (34) (36). Also consider given quadratic Lyapunov performance requirements (7) and let Assumptions 1 , 2 , 3 hold. Then almost surely with respect to the channel sequence $\left\{h_{k}, k \geq 0\right\}$ for any $\epsilon>0$ there exists a time slot $K$ such that for any $k \geq K$ the control performances satisfy

$$
\begin{aligned}
\mathbb{E}\left[V_{i}\left(x_{i, k+1}\right) \mid\right. & \left.x_{i, k}, h_{0}, \ldots, h_{k-1}\right] \\
& \leq\left(\rho_{i}+\epsilon\right) V_{i}\left(x_{i, k}\right)+\operatorname{Tr}\left(P_{i} W_{i}\right),
\end{aligned}
$$

and the power consumption satisfies

$$
\mathbb{E}\left[\sum_{i=1}^{m} \alpha_{i, k} p_{i, k} \mid x_{i, k}, h_{0}, \ldots, h_{k-1}\right] \leq P+\epsilon
$$

where $P$ is the optimal value of the optimization problem (10).

\section{Proof. See Appendix A.3}

According to the theorem after a sufficiently large time horizon the scheduling and power allocation variables obtained online by the proposed stochastic algorithm reach the optimal performance by (15). In particular the Lyapunov requirements of all control systems are satisfied in the limit and the optimal power expenditure is reached. Note however that the theorem does not provide any guarantee on how fast the solution converges to the optimal one. We discuss this issue along with other limitations of the algorithm in Section 6 , In the following section we present simulations verifying our theoretical results, and also indicating that the convergence of the algorithm is relatively fast enough so that online control performance is not severely affected.

\section{SIMULATIONS}

In this section we illustrate the advantages of the proposed opportunistic resource allocation methodology in wireless NCSs. Recall that by solving the auxiliary problems (14). control systems with vector states are equivalently converted into scalar constraints in (15). Hence without loss of generality we examine scalar control systems.

Consider a heating system application controlling the temperature in two independent rooms of a building. Assuming the wireless NCS architecture of Fig. 1 with $m=2$, wireless sensors transmit the temperatures of each room to a central location (access point) responsible for adjusting the heating in the rooms. For simplicity suppose both systems have identical dynamics and are of the form (1) with state $x_{i, k}$ denoting the difference between current and desired temperature for room $i$. In particular suppose that when system $i$ transmits $\left(\gamma_{i, k}=1\right)$, heating is activated for system $i$ and results in stable dynamics $A_{c, i}=0.4$ in (1) Otherwise if $\gamma_{i, k}=0$ the system is open loop unstable with $A_{o, i}=1.1$ in (1), e.g., because heating is deactivated.

For symmetry let also channel states $h_{1, k}$ and $h_{2, k}$ be independent for each system, both having an exponential distribution with mean 1 . The function $q(h, p)$ is shown in Fig. 2. For these scalar systems it suffices to consider Lyapunov functions $V_{i}(x)=x^{2}$. We require then that system 1 guarantees a high Lyapunov decrease $\rho_{1}=0.75$ rate according to (7), while system 2 only requires $\rho_{2}=0.90$. In this case system 1 has a higher transmission success requirement $c_{1} \approx 0.44$ by $(14)$ compared to $c_{2} \approx 0.30$ of system 2 .

After solving problem (15) offline according to the subgradient method of Section 3 the optimal channel-aware scheduling and power allocation variables are depicted in Fig. 3 and Fig. 4 respectively. As we observe in Fig. 3, System 1 which requires higher transmission success $c_{1}$ is scheduled to transmit for most values of the observed channel pair $\left(h_{1}, h_{2}\right)$. System 2, which has a lower control requirement, is scheduled only if its channel $h_{2}$ is sufficiently favorable and system 1 experiences an adverse channel $h_{1}-$ see Fig. 3. This illustrates how the opportunistic scheduler exploits channel conditions to select which system will close the loop to meet the Lyapunov constraints. Also, as we will see next, when both systems experience very adverse channels, scheduling is irrelevant because the optimal transmit powers then are zero (no transmission).

The optimal transmit power allocation is decentralized as we noted in Remark 2, i.e., $p_{i}$ only depends on the channel $h_{i}$ system $i$ experiences, and thus we plot in Fig. 4 power allocation for both systems on same axes. System 1, which has a more demanding control constraint, requires in general higher transmit power since it is scheduled to transmit even under adverse channel states as we saw in Fig. 3. This is also 


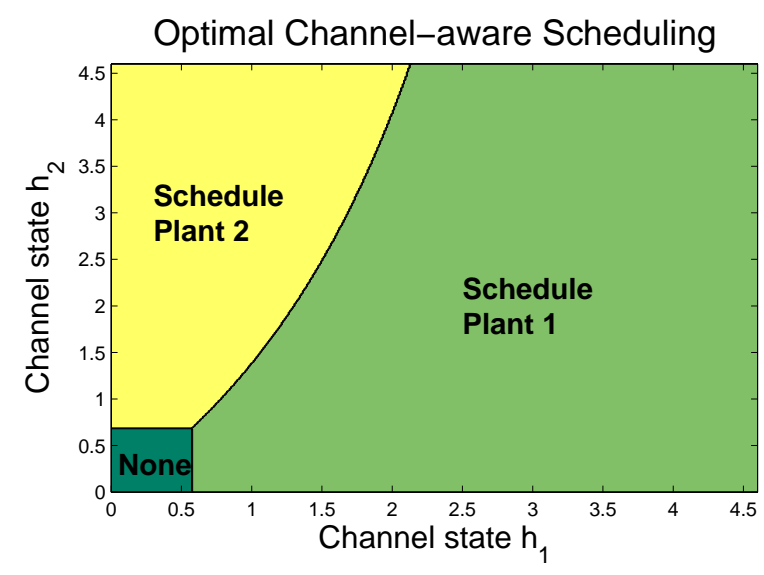

Figure 3: Optimal channel-aware scheduling for the example presented in simulations. System 1 has a harder Lyapunov decrease rate requirement and is scheduled to transmit for most observed channel states $h_{1}, h_{2}$. System 2 is scheduled only if its channel conditions $h_{2}$ are much more favorable that those of system 1 . When both channels are very adverse systems select zero transmit powers so scheduling is irrelevant.

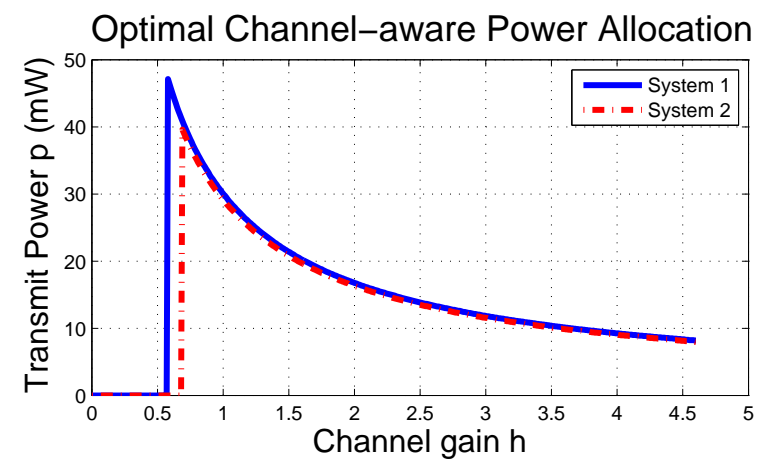

Figure 4: Optimal channel-aware power allocation for the example presented in simulations. Under adverse channel conditions systems do not transmit. The channel threshold for transmission for system 1 is lower than that of system 2 because the former has a higher Lyapunov decrease rate requirement. System 1 also requires higher transmit power.

captured in the expected power consumption $\mathbb{E}_{h} \alpha_{i}(h) p_{i}^{*}\left(h_{i}\right)$ of each system computed numerically as $\approx 11.2 \mathrm{~mW}$ for system 1 and $\approx 6.7 \mathrm{~mW}$ for system 2 .

To demonstrate the power savings of the opportunistic resource allocation we compare to a simple non-channel-aware communication design. In particular suppose that when systems are scheduled to transmit they select a constant transmit power equal to the value $p_{\max }=75 \mathrm{~mW}$ resulting in a probability of success $\mathbb{E}_{h_{i}} q\left(h_{i}, p_{\max }\right) \approx 0.74$. To guarantee the Lyapunov requirements $c_{i}$ the scheduler must select constant

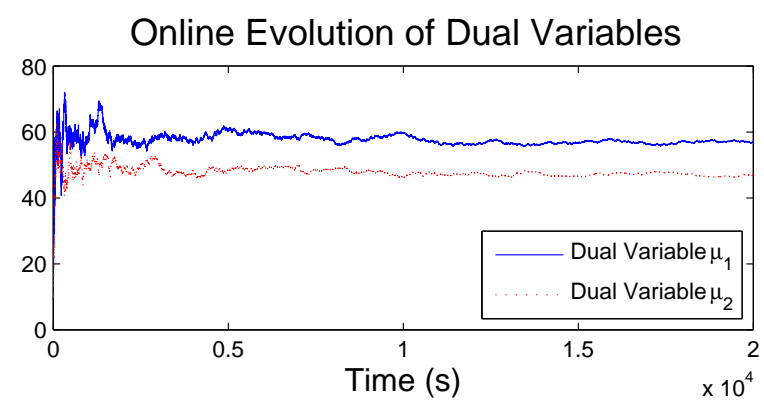

Figure 5: After a number of steps during the online algorithm the dual variables $\mu_{k}$ remain in a neighborhood around the optimal $\mu^{*}$.

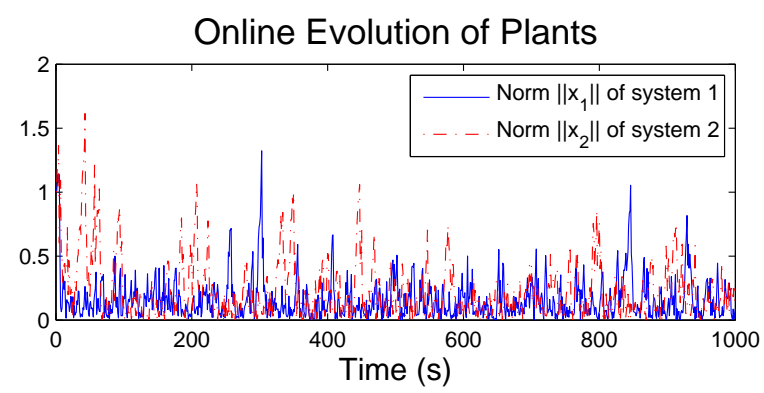

Figure 6: Magnitude of the control system states $\left\|x_{1, k}\right\|$, $\left\|x_{1, k}\right\|$ during the first steps of the online algorithm.

values $\alpha_{1} \approx 0.6, \alpha_{2} \approx 0.4$ leading to total power consumption $\left(\alpha_{1}+\alpha_{2}\right) p_{\max } \approx 75 \mathrm{~mW}$, significantly higher than the total $17.9 \mathrm{~mW}$ of the opportunistic case. Hence in this example the opportunistic resource allocation succeeded almost a $76 \%$ decrease in power consumption.

Then, for the same system parameters and Gaussian process noise $w_{i, k}$ with standard deviation $\sqrt{W_{i}}=0.1$, we implement the stochastic online algorithm of Section 4 given by (34) (36). The evolution of the dual variables $\mu_{k}$ during the simulation is shown in Fig. 5. After some time (here steps $k$ correspond to seconds) the dual iterates remain in a small neighborhood around the optimal $\mu^{*}$, which agrees with the theoretical a.s. convergence of Prop. 3. Equivalently the scheduling and power allocation decisions taken online after a number of iterations are almost feasible for the constraints of problem (15). The plant states of both systems remain bounded during execution, as we show by simulations in Fig. 6. The empirical average quadratic costs $1 / N \sum_{k=1}^{N} V_{i}\left(x_{i, k}\right)$ were respectively 0.047 for system 1 and 0.088 for system 2 , matching closely the constant value $\operatorname{Tr}\left(P_{i} W_{i}\right) /\left(1-\rho_{i}\right)$ of the theoretical limit quadratic costs by (8) which were 0.04 and 0.1 respectively.

\section{DISCUSSION AND CONCLUSIONS}

In this paper we present a framework for designing opportunistic channel-aware schedulers for NCSs closing their 
loops over a shared wireless medium. We formulate the scheduling and the transmit power design problem in a stochastic optimization framework where the objective is to minimize the total expected power expenditures while guaranteeing that given Lyapunov functions for each of the control systems exhibit a desired decrease rate. We presented an offline optimization algorithm, as well as an online stochastic algorithm utilizing the random observed channel sequence to solve the problem.

While the proposed online algorithm guarantees almost sure convergence to the optimal solution, it does not provide a characterization of the convergence rate. This could potentially introduce a long transient control system behavior before the desired performance is reached. Another drawback is that the online algorithm uses decreasing step sizes, which limits the ability to adapt to an environment where the channel distributions are not stationary but vary with time. These issues will be the focus of future work. An altogether different research direction is to include the measured plant system states in the scheduling decisions, similarly to the power management paradigm for a single control loop in [6, 7].

\section{APPENDIX}

\section{A. PROOFS OF STATEMENTS}

\section{A.1 Proof of Lemma 1}

The variables $\alpha, p$ minimizing the Lagrangian at the form (30) for some $\mu \in \mathbb{R}_{+}^{m}$ solve

$$
\min _{\alpha \in \mathcal{A}, p \in \mathcal{P}} \mathbb{E}_{h} \sum_{i=1}^{m} \alpha_{i}(h)\left[p_{i}(h)-\mu_{i} q\left(h_{i}, p_{i}(h)\right)\right] .
$$

Without loss of generality we can exchange the expectation and the minimization over $\alpha(),. p($.$) in (40) to solve$

$$
\min _{\alpha(h), p(h)} \sum_{i=1}^{m} \alpha_{i}(h)\left[p_{i}(h)-\mu_{i} q\left(h_{i}, p_{i}(h)\right)\right] .
$$

This is valid because any pair $\alpha(),. p($.$) that does not min-$ imize the objective in (41) on a set of variables $h$ with $\phi$ positive measure must yield a strictly larger expected value in the objective of (40). In other words, the minimizers of (40) can only differ from the minimizers of (41) at a set of values $h$ with measure zero. Note then that $\alpha_{i}(h) \geq 0$ always so that (41) can be rearranged to

$$
\min _{\alpha(h)} \sum_{i=1}^{m} \alpha_{i}(h) \min _{p_{i}(h)} p_{i}(h)-\mu_{i} q\left(h_{i}, p_{i}(h)\right) .
$$

This corresponds exactly to solving (31) (32)

Now let us prove the second statement of the lemma. We need to show that any pair $(\alpha(\mu), p(\mu))$, which are functions of $h$, solving (40) gives a unique evaluation of $s(\mu)$, where by (25) $s_{i}(\mu)$ involves integrating

$$
\alpha_{i}(\mu ; h) q\left(h_{i}, p_{i}(\mu ; h)\right)
$$

with respect to distribution $\phi$ of $h$. Hence it suffices to show that (43) is unique $\phi$-a.s. We argued that (40) is a.s. equivalent to (41), which is equivalent to (42) Note that in the special case where $\alpha_{i}(\mu ; h)=0$ for some $i$ the choice $p_{i}(\mu ; h)$ does not matter in $s_{i}(\mu)$ since (43) equals zero.

Hence we only need to show that the minimizers $\alpha(\mu ; h)$, $p(\mu ; h)$ of (42) imply a.s. uniqueness of (43). By Assumption 2(c) the minimizer $p(\mu ; h)$ by $(31)$ is a.s. unique so we only examine $\alpha(\mu ; h)$ by (32) For any vector $h$ that has a unique minimizer $j=\operatorname{argmin}_{i} \xi\left(h_{i}, \mu_{i}\right)$ it follows immediately that $\alpha_{j}(\mu ; h)=1$ is the unique optimal. Thus we are left to examine the case of multiple $\operatorname{argmin}_{i} \xi\left(h_{i}, \mu_{i}\right)$, in which case $\alpha(h)$ minimizing (32) is not unique, and show that a.s. uniqueness of (43) is satisfied.

First, if for some $h$ there are multiple $\operatorname{argmin}_{i} \xi\left(h_{i}, \mu_{i}\right)$ satisfying $\xi\left(h_{i}, \mu_{i}\right)=0$, by Assumption 2(c) almost surely the minimizer in (33) is unique. Note also that $p_{i}(\mu ; h)=0$ for all such $i$ must be a minimizer, because it gives an objective for problem (33) equal to $0-\mu_{i} q\left(h_{i}, 0\right)=0$ by Assumption 2(a). Hence $p_{i}(\mu ; h)=0$ is almost surely the unique minimizer and in that case $q\left(h_{i}, p_{i}(\mu ; h)\right)=0$, and any choice $\alpha(h)$ gives a unique value of (43), equal to 0 .

Finally we argue that the event where more than one minimizers $j, k \in \operatorname{argmin}_{i} \xi\left(h_{i}, \mu_{i}\right)$ exist with $\xi\left(h_{j}, \mu_{j}\right)=$ $\xi\left(h_{k}, \mu_{k}\right)<0$ has probability zero. To do so we make use of the following fact.

Fact: If $\xi\left(h_{i}, \mu_{i}\right)<0$, it must be that the argument $p_{i}(\mu ; h)$ by (31) of the minimization (33) satisfies $q\left(h_{i}, p_{i}(\mu ; h)\right)>$ 0 . This is true because otherwise we would have $\xi\left(h_{i}, \mu_{i}\right) \geq$ 0 . Also by Assumption 2(b) when $q()>$.0 , it is strictly increasing in its argument. Thus we have for $h_{i}^{\prime}>h_{i}$ that

$$
\begin{aligned}
\xi\left(h_{i}, \mu_{i}\right) & =p_{i}(\mu ; h)-\mu_{i} q\left(h_{i}, p_{i}(\mu ; h)\right) \\
& >p_{i}(\mu ; h)-\mu_{i} q\left(h_{i}^{\prime}, p_{i}(\mu ; h)\right) \geq \xi\left(h_{i}^{\prime}, \mu_{i}\right) .
\end{aligned}
$$

This means that for a fixed $\mu_{i}$, when $\xi\left(h_{i}, \mu_{i}\right)$ is negative it is strictly decreasing as the argument $h_{i}$ increases.

By the fact above, when $\xi\left(h_{j}, \mu_{j}\right)$ is negative it is strictly decreasing in the argument $h_{j}$. Therefore for fixed $\mu$ the event that $\left\{h_{j}, h_{k}: \xi\left(h_{j}, \mu_{j}\right)=\xi\left(h_{k}, \mu_{k}\right)<0\right\}$ defines at most a (one dimensional) line on the set $\mathcal{H}^{2}$ of possible values for $h_{j}, h_{k}$. By Assumption $1 \phi$ has a density on $\mathcal{H}^{m}$, therefore it assigns zero measure on any lower-dimensional subset of $\mathcal{H}^{m}$. So the event of multiple equal and negative $\xi\left(h_{i}, \mu_{i}\right)$ has probability zero, and does not affect the a.s. uniqueness of (43)

\section{A.2 Proof of Proposition 3}

The proof relies on a supermartingale convergence argument frequently used in stochastic optimization - see, e.g., [14]. First note that for any $k$ the vector $s_{k}$ by (35) satisfies

$$
g\left(\mu^{\prime}\right)-g\left(\mu_{k}\right) \leq\left(\mu^{\prime}-\mu_{k}\right)^{T} \mathbb{E}\left[s_{k} \mid \mu_{k}\right]
$$

for all $\mu^{\prime} \in \mathbb{R}_{+}^{m}$. To show this fact compare (34) (35) with (25) to conclude that $\mathbb{E}\left[s_{k} \mid \mu_{k}\right]=s\left(\mu_{k}\right)$ because $h_{k}$ is i.i.d for every $k$. Inequality (45) then follows directly from (24) 
By Assumption 3 there exists a strictly feasible primal solution $\alpha^{\prime}(),. p^{\prime}($.$) . Call P^{\prime}$ the resulting objective value of (15) and let $\epsilon^{\prime}>0$ denote the constraint slack of (17) i.e., $c_{i}+\epsilon^{\prime} \leq \mathbb{E}_{h} \alpha_{i}^{\prime}(h) q\left(h_{i}, p_{i}^{\prime}(h)\right)$. Then we may bound the dual function (19) at any point $\mu$ by

$g(\mu) \leq L\left(\alpha^{\prime}, p^{\prime}, \mu\right)=P^{\prime}+\sum_{i=1}^{m} \mu_{i}\left[c_{i}-\mathbb{E}_{h} \alpha_{i}^{\prime}(h) q\left(h_{i}, p_{i}^{\prime}(h)\right)\right]$

which by the assumption is upper bounded by $P^{\prime}-\sum_{i=1}^{m} \mu_{i} \epsilon^{\prime}$. Rearranging terms, and since $\mu \geq 0$ it follows that for every $i, \mu_{i} \leq \sum_{j=1}^{m} \mu_{j} \leq\left(P^{\prime}-g(\mu)\right) / \epsilon^{\prime}$. In particular we find that the optimal dual variables are finite, $\mu_{i}^{*} \leq\left(P^{\prime}-D\right) / \epsilon^{\prime}$.

Since the optimal dual variables are finite, the distance $\left\|\mu_{k}-\mu^{*}\right\|$ is a well-defined and bounded random variable depending on the random $\mu_{k}$ obtained by (36) The following lemma examines how this distance evolves. Recall that as we commented after (25) the subgradients $s(\mu)$ are always bounded in our problem.

LEMMA 2. Let $D$ be the optimal value of the dual problem (21) $\mu^{*}$ be an optimal solution, and $S$ be the bound on the subgradient $\|s(\mu)\| \leq S$ for any $\mu \in \mathbb{R}_{+}^{m}$. Then at each step $k$ of the update (36) we have

$\mathbb{E}\left[\left\|\mu_{k+1}-\mu^{*}\right\|^{2} \mid \mu_{k}\right] \leq\left\|\mu_{k}-\mu^{*}\right\|^{2}+\epsilon_{k}^{2} S^{2}-2 \epsilon_{k}\left(D-g\left(\mu_{k}\right)\right)$

PROOF. First use the expression for $\mu_{k+1}$ in (36) to write $\left\|\mu_{k+1}-\mu^{*}\right\|=\left\|\left[\mu_{k}+\epsilon_{k} s_{k}\right]_{+}-\mu^{*}\right\| \leq\left\|\mu_{k}+\epsilon_{k} s_{k}-\mu^{*}\right\|$,

where the last inequality holds because projecting on the positive orthant can only decrease the distance from a point $\mu^{*}$ in the orthant. Taking expectation on both sides given $\mu_{k}$ and expanding the square norm of the right hand side, we get

$$
\begin{aligned}
\mathbb{E}\left[\left\|\mu_{k+1}-\mu^{*}\right\|^{2} \mid \mu_{k}\right] \leq & \left\|\mu_{k}-\mu^{*}\right\|^{2}+\epsilon_{k}^{2} S^{2} \\
& +2 \epsilon_{k}\left(\mu_{k}-\mu^{*}\right)^{T} \mathbb{E}\left[s_{k} \mid \mu_{k}\right]
\end{aligned}
$$

where we bounded $\left\|\mathbb{E}\left[s_{k} \mid \mu_{k}\right]\right\|^{2}<S^{2}$. The statement (47) follows from (49) by applying inequality (45) with the substitution $\mu^{\prime}=\mu^{*}$.

Our goal is to use (47) to show that $\left\|\mu_{k+1}-\mu^{*}\right\|^{2} \rightarrow 0$ almost surely. First note that at any $\mu_{k}$ the dual function is lower than the optimal value (cf. (21), so $D-g\left(\mu_{k}\right) \geq 0$. Hence (47) can be simplified to

$$
\mathbb{E}\left[\left\|\mu_{k+1}-\mu^{*}\right\|^{2} \mid \mu_{k}\right] \leq\left\|\mu_{k}-\mu^{*}\right\|^{2}+\epsilon_{k}^{2} S^{2} .
$$

Then define the non-negative random variable

$$
a_{k}=\left\|\mu_{k}-\mu^{*}\right\|^{2}+\sum_{\ell=k}^{\infty} \epsilon_{l}^{2} S^{2}
$$

which depends on the sequence (filtration) $\mathcal{F}_{k}=\left\{\mu_{0}, \ldots, \mu_{k}\right\}$. Note that $a_{k}$ is bounded because $\mu_{k}$ generated by (36) is bounded at every $k$ and also by assumption the stepsizes are square summable. Hence $a_{k}$ is integrable. By the relation (50) it easily follows that $a_{k}$ satisfies

$$
\mathbb{E}\left[a_{k+1} \mid \mathcal{F}_{k}\right] \leq a_{k} .
$$

Such a stochastic process, i.e., integrable, adapted to $\mathcal{F}_{k}$, and satisfying (52) is called a supermartingale [4, Ch. 5]. Moreover, a non-negative supermartingale converges almost surely to some limit random variable [4, Th. 5.2.9]. Observe that the second summand $\sum_{\ell=k}^{\infty} \epsilon_{l}^{2} S^{2}$ of $a_{k}$ in (51) is deterministic and converges to 0 because of square summability of the stepsizes. Hence the random variable $\left\|\mu_{k}-\mu^{*}\right\|^{2}$ converges almost surely (to some random variable).

To arrive at a contradiction suppose the limit random random variable is not identically zero, i.e., it takes positive values with nonzero probability. Equivalently there exist $\delta>0$ and $\epsilon>0$ such that with probability $\delta$ we have $\left\|\mu_{k}-\mu^{*}\right\| \geq \epsilon$ for all sufficiently large $k$. This implies that $\mu_{k}$ are bounded away from the optimal, i.e., that for sufficiently large $k$ we have $D-g\left(\mu_{k}\right) \geq \epsilon^{\prime}$ for some $\epsilon^{\prime}>0$. Hence with probability $\delta$ we have $\sum_{k=0}^{\infty} 2 \epsilon_{k}\left(D-g\left(\mu_{k}\right)\right)=+\infty$. This implies

$$
\mathbb{E} \sum_{k=0}^{\infty} 2 \epsilon_{k}\left(D-g\left(\mu_{k}\right)\right)=+\infty
$$

which however contradicts with (47). The reason is that if we take expectation on both sides of (47) and iterate for $k=$ $0, \ldots, N$ we get

$$
\begin{aligned}
0 & \leq \mathbb{E}\left\|\mu_{N+1}-\mu^{*}\right\|^{2} \leq\left\|\mu_{0}-\mu^{*}\right\|^{2} \\
& +\sum_{k=0}^{N} \epsilon_{k}^{2} S^{2}-\mathbb{E} \sum_{k=0}^{\infty} 2 \epsilon_{k}\left(D-g\left(\mu_{k}\right)\right) .
\end{aligned}
$$

The right hand side then needs to be positive, but (53) implies that in the limit as $N \rightarrow \infty$ the right hand side becomes negative. Therefore it must happen that $\left\|\mu_{k}-\mu^{*}\right\|$ converges to zero with probability 1.

By continuity of the (concave) dual function $g(\mu)$ we also have that $g\left(\mu_{k}\right)$ converges to the optimal $g\left(\mu^{*}\right)=D$ a.s.

\section{A.3 Proof of Theorem 1}

First note that by imitating the steps leading from $(10)$ to (15) the statement of (38) becomes equivalent to

$$
c_{i}-\mathbb{E}_{h_{k}}\left[\alpha_{i, k} q\left(h_{i, k}, p_{i, k}\right) \mid \mu_{k}\right] \leq \epsilon^{\prime},
$$

for some appropriate constant $\epsilon^{\prime}>0$. To suppress notation we have exploited the fact that according to the online algorithm the variables $\alpha_{k}, p_{k}$ depend just on the value of $\mu_{k}$ and not on the whole observed channel history. Also by the expression of $s_{k}$ given in (35) equation (55) is equivalent to $\mathbb{E}_{h_{k}}\left[s_{k} \mid \mu_{k}\right] \leq \epsilon^{\prime}$. Then as we argued in the proof of Prop. $3 \mathbb{E}_{h_{k}}\left[s_{k} \mid \mu_{k}\right]=s\left(\mu_{k}\right)$ where $s\left(\mu_{k}\right)$ is given by $(25)$. To sum up, we have shown so far that (38) is equivalent to $s\left(\mu_{k}\right) \leq \epsilon^{\prime}$.

Under Assumption 3 we have established in Proposition 3 that for the online algorithm $\mu_{k} \rightarrow \mu^{*}$ almost surely with respect to the channel sequence $\left\{h_{k}, k \geq 0\right\}$. Then we note 
a convex analysis fact by [1, Prop. 4.2.3]. If $g$ is concave, and $\mu_{k} \rightarrow \mu^{*}$, and $s\left(\mu_{k}\right)$ is selected as a subgradient of $g$ at $\mu_{k}$, then every limit point of $s\left(\mu_{k}\right)$ is a subgradient of $g$ at $\mu^{*}$. Hence for the sequence of $\mu_{k}$ obtained by the online algorithm we have that almost surely the sequence $s\left(\mu_{k}\right)$ converges to a subgradient of $g$ at $\mu^{*}$.

Then under Assumptions 1, 2, 3 we can combine Prop. 1 (c) with Lemma 1 to conclude that all subgradients at $\mu^{*}$ satisfy $s\left(\mu^{*}\right) \leq 0$ - a detailed argument was given preceding Prop. 2] Hence for the sequence of $\mu_{k}$ obtained by the online algorithm we have that almost surely limsup $s\left(\mu_{k}\right) \leq 0$. This proves statement (38) via the equivalence we established above between $s\left(\mu_{k}\right) \leq \epsilon^{\prime}$ and (38).

Finally let us prove (39), Recall that the dual function equals $g(\mu)=L(\alpha(\mu), p(\mu), \mu)$ where $\alpha(\mu), p(\mu)$ are Lagrange optimizers at $\mu$ according to (20). Using the definition of the Lagrangian at (18) and the interpretation of the subgradient $s(\mu)$ at $(25)$ as the constraint slack, we have that for any $\mu_{k}$

$$
g\left(\mu_{k}\right)=\mathbb{E}_{h} \sum_{i=1}^{m} \alpha_{i}\left(\mu_{k} ; h\right) p_{i}\left(\mu_{k} ; h\right)+\mu_{k}^{T} s\left(\mu_{k}\right)
$$

Now observe that the expectation in (39) equals the expectation given in (56) because by step (34) the primal variables are selected as Lagrange optimizers at $\mu_{k}$. Therefore to show that (39) holds a.s. it suffices to show that the expectation in (56) converges a.s. to $D$, which equals $P$ by duality.

Proposition 3 establishes that the left hand side of (56) satisfies $g\left(\mu_{k}\right) \rightarrow D$, and also that $\mu_{k} \rightarrow \mu^{*}$ a.s. We have also already argued that $s\left(\mu_{k}\right) \rightarrow s\left(\mu^{*}\right)$ a.s. Therefore also $\mu_{k}^{T} s\left(\mu_{k}\right) \rightarrow \mu^{* T} s\left(\mu^{*}\right)$ a.s. But by Prop. 1 (b) $\mu^{* T} s\left(\mu^{*}\right)=0$. This completes the proof.

\section{B. REFERENCES}

[1] D. P. Bertsekas, A. Nedić, and A. E. Ozdaglar. Convex analysis and optimization. Athena Scientific, 2003.

[2] M. S. Branicky, S. M. Phillips, and W. Zhang. Scheduling and feedback co-design for networked control systems. In Proc.of the 41st IEEE Conference on Decision and Control, 2002, volume 2, pages 1211-1217, 2002.

[3] A. Cervin, J. Eker, B. Bernhardsson, and K.-E. Årzén. Feedback-feedforward scheduling of control tasks. Real-Time Systems, 23(1-2):25-53, 2002.

[4] R. Durrett. Probability: theory and examples. Cambridge University Press, 2010.

[5] M. Egerstedt and Y. Wardi. Multi-process control using queuing theory. In Proc. of the 41st IEEE Conference on Decision and Control, volume 2, pages 1991-1996, 2002.

[6] K. Gatsis, M. Pajic, A. Ribeiro, and G. J. Pappas. Power-aware communication for wireless sensor-actuator systems. In Proc. of the 52nd IEEE Conference on Decision and Control (CDC 2013),
2013. To appear.

[7] K. Gatsis, A. Ribeiro, and G. J. Pappas. Optimal power management in wireless control systems. In Proc. of the 2013 American Control Conference (ACC 2013), pages 1562-1569, 2013.

[8] L. Georgiadis, M. J. Neely, and L. Tassiulas. Resource allocation and cross-layer control in wireless networks. Now Publishers Inc, 2006.

[9] A. Goldsmith. Wireless communications. Cambr. Univ. Press, 2005.

[10] D. Hristu-Varsakelis. Feedback control systems as users of a shared network: Communication sequences that guarantee stability. In Proc. of the 40th IEEE Conference on Decision and Control, 2001, volume 4, pages 3631-3636, 2001.

[11] J. Le Ny, E. Feron, and G. J. Pappas. Resource constrained lqr control under fast sampling. In Proc. of the 14th international conference on Hybrid systems: computation and control, pages 271-280. ACM, 2011.

[12] X. Liu, E. K. Chong, and N. B. Shroff. A framework for opportunistic scheduling in wireless networks. Computer Networks, 41(4):451-474, 2003.

[13] H. Rehbinder and M. Sanfridson. Scheduling of a limited communication channel for optimal control. Automatica, 40(3):491-500, 2004.

[14] A. Ribeiro. Optimal resource allocation in wireless communication and networking. EURASIP Journal on Wireless Communications and Networking, 2012(1):1-19, 2012.

[15] G. Weiss and R. Alur. Automata based interfaces for control and scheduling. In Hybrid Systems: Computation and Control, pages 601-613. Springer, 2007.

[16] L. Zhang and D. Hristu-Varsakelis. Communication and control co-design for networked control systems. Automatica, 42(6):953-958, 2006.

[17] W. Zhang, M. Branicky, and S. Phillips. Stability of networked control systems. IEEE Control Systems Magazine, 21(1):84-99, 2001. 